\title{
https://doi.org/10.46344/JBINO.2020.v09i5b.09
}

\section{CRITICAL REVIEW AND ANALYSIS OF NEPHRO-PROTECTIVE DRUGS IN AYURVEDA}

\section{Eknath G. Kulkarni', Sunil H. Pal2 \& Japa J. Phadke ${ }^{3}$}

'Associate Professor, Department of Kayachikitsa,

2MD Scholar, Department Of Kayachikitsa

3MD Scholar, Department Of Kayachikitsa

A.S.S. Ayurved Mahavidyala Arogyashala Rugnalaya, Panchvati, Nashik-422003

Email ID- sunilp175@gmail.com

\begin{abstract}
Medicinal plants may serve as a vital source of potentially useful new compounds for the development of effective therapy to combat a varieties of Kidney problems. Many herbs have been proven to be effectual as Nephro-protective agents. Developing a satisfactory herbal therapy to treat severe renal disorders, requires systematic investigation of properties like Acute Renal Failure (ARF), Nephrotic syndrome and chronic interstitial nephritis. Chronic Renal Failure (CRF) is considered when glomerular filtration rate (GFR) falls below $30 \mathrm{ml} / \mathrm{min}$. The conventional approach of management includes Dialysis and Renal Replacement Therapy (RRT) which are not affordable by Indian Populations due to economic status. Therefore, exploration of a safe and alternative therapy is needed. In Ayurveda, some of the herbs possess Nephro-protective properties due to presence of their chemical components like Kanchnar (Bauhinia variegate)l, Kushmanda (Benincasa hispida), Yeshtimadhu (Glycyrrhiza glabra). The present review is aimed to elucidate the list of Nephro-protective medicinal plants, which are scientifically proved in treating renal disorders.
\end{abstract}

Keywords-Kidney disease, Ayurveda, Nephro-protective 


\section{Introduction}

Nephrotoxicity is one of the most commonly kidneys problems and occurs when body is exposed to a drug toxicity[1].A number of therapeutic agents can adversely affect the kidney, resulting in Acute Renal Failure (ARF),Chronic interstitial nephritis and nephrotic syndrome. There is an increasing number of potent therapeutic drugs like aminoglycosides antibiotics,Non-steroidal antiinflammatory drugs, Chemotherapeutic agents have been added to the therapeutic arsenal in recent years [2]. Exposure to chemical reagents like ethylene glycol, carbon tetra chloride, sodium oxalate and heavy metals such as lead,mercury, cadmium, arsenic includes nephrotoxicity. Prompt recognition of the disease and cessation of responsible drugs are usually the only necessary therapy [3].Nephroprotective agents are the drugs which possess protective activity against nephrotoxicity. Medicinal plants have curative properties due to presence of various complex chemical substance. Early literatures have prescribed various herbs for the cure of renal disorders [4].The term renal failure primarily denotes failure of the excretory function of kidneys, leading to retention of nitrogenous waste products of metabolism in the blood [5].In addition to this, there is a failure of regulation of fluid and electrolyte balance along with endocrine dysfunction. The renal failure is fundamentally categorized into acute and chronic renal failure [6].

Acute Renal Failure (ARF) refers to the sudden and usually reversible loss of renal function, which develops over a period of days or weeks. There are many causes for Acute Renal Failure(ARF) which mainly includes Acute Tubular Necrosis (ATN) that commonly occurs for $85 \%$ of incidence. Mostly acute tubular necrosis occurs either due to exogenous or endogenous. The exogenous agents are radio-contrast agents, cyclosporine, antibiotics, chemotherapeutic agents $[5,6]$. Chronic Renal Failure (CRF) is an irreversible deterioration in the renal function which classically develops over a period of years, leading to loss of excretory metabolic and endocrine functions, various causes of renal failure has been recognized like Hypertension, Diabetes mellitus, Antineoplastic agents like

cyclophosphamide, vincristine, cisplatin etc. [5]

In Ayurveda, Chronic Kidney Disease (CKD) described as a mootra dosha vikara and causing an edema. Both kidney are root of medovaha srotas[7].According to Acharya Charaka the causes of mootra dosha vikara are vitiated by the intake of unhealthy drinks and foods, sexual intercourse while having the urge of micturition, disorder of electrolyte imbalance, malnutrition, and severe traumatic injury [7].

\section{Pathogenesis Of Kidney Disease In Ayurveda}

The pathogenesis of kidney diseases is not separately mentioned. It can be included in Prameha, Mootra dosha, Mootra kriccha, injury of vankshana, Ashmari and Shotha etc. In Charaka Samhita, described that kidney and bladder are the root (controlling organ) of the channels carrying urine and fat, mamsa and liquid dhatus of the body. The vitiated doshas while coming in contact with the opening of these channels obstruct them. This result in the manifestation of kidney disease. While become chronic or incurable due to the affection of all the qualities of doshas and also due to the simultaneous vitiation of dhatus [8].

\section{Agents which Causes Nephrotoxicity}

Drugs, diagnostic agents and chemicals (fertilizers, pesticides) are well known to be nephrotoxic. The following are some of the important nephrotoxic agents [9]. 

A) Heavy
metal-
Mercury,Arsenic, Lead
B) Anti-neoplastic agents
a) Ankylating-agents-Cisplatin, cyclophosphamide
b) Nitrosoureas-Carmustine
c) Anti-Tumour
Doxorubicin
C) Anti-microbial-
Tetracycline,Acyclovir,Trimethoprin, Rifampicin,Amphotericin-B
D) Aminoglycosides-
Gentamycin,Amikacin,Kanamycin, Streptomycin
E) Misc.
a) Radio-contrast-agents-
NSAID's, Ibuprofen,Indomethaci $n$, Aspirin

\section{Concept Of Nephro-protective Drugs in Ayurveda}

In Ayurveda text, some herbs are mentioned having specific actions on Mutravaha srotas (Urinary System) and its diseases. These herbs are classified under Mutravirechaneeya Gana are Diuretic in action and Mutraviranjaneeya Gana acts on removal of dushita doshas from urine [7].

\section{Materials and Methods-}

\section{Materials -}

Data are collected from various classical Samhita of Ayurveda and their commentaries, modern medicine books, published research articles, paper, journals and internet.

\section{Methods -}

Type of study: - Critico-conceptual study

\section{Plants with Nephroprotective effects-}

The following plants are having Nephroprotective activity

\section{Bauhinia variegate (Kanchnar)}

Nephroprotective activity of the ethanolic and aqueous extracts of root of Bauhinia variegata at a dose of 400 $\mathrm{mg} / \mathrm{kg}$ bw was evaluated by gentamicin and cisplatin induced nephrotoxicity in rats. Both extracts showed nephroprotective activity in both gentamicin and cisplatin induced nephrotoxicity models as evident by decrease in serum creatinine, serum urea, urine creatinine and BUN levels in extract treated groups which was elevated by gentamicin and cisplatin in the respective models, which also confirmed by histopathological study [10-11].

\section{Benincasa hispida (Kushmanda)}

Hydro-alcoholic extract of Benincasa hispida whole fruit extract significantly increased the tissue GSH levels and reduced lipid peroxidation levels. Furthermore, it was confirmed by the histopathological observation that the degenerative changes caused by paracetamol were also restored by treatment with hydro-alcoholic extract of Benincasa hispida whole fruit extract [78]. It was also produced nephroprotective activity against mercury poisoning in rats [12-13].

\section{Tribulus terrestris (Gokshura)}

Diuretics-Rich in potassium and nitrates[14]

\section{Brassica nigra (Rajkshavak)}

The crude methanol extract of Brassica nigra leaf lacks inherent toxicity and exhibits hepatic and nephroprotective [15-16].

\section{Brassica rapa}

The effect of the ethanol extract of the roots of Brassica rapa (EBR) to ameliorate cisplatin-induced nephrotoxicity was studied in terms of oxidative stress, as characterized by lipid peroxidation, reactive oxygen species (ROS) production, and glutathione (GSH) depletion in LLC-PK 1 cells [17]. 


\section{Bryophyllum calycinum}

The aqueous extract of the leaves possessed potent nephroprotective activity in gentamycin-induced nephrotoxicity in rats. The plant hydroalcoholic extract was also found to exert significant diuresis and antiurolithitic activity when given by oral and ip route to rats [18-21].

\section{Carum carvi (Krishna Jeeraka)}

The renoprotective effect of aqueous extract of Carum carvi seeds was evaluated in experimentally induced diabetic nephropathy (DN) in rodents. The morphological examination of untreated diabetic rats kidneys showed glomerular and tubular degeneration with massive cellular infiltration, hemorrhage in interstitial tissue and deformed renal tissue architecture. Whereas the kidney of Carum carvi essential oil treated rats showed marked improvement with minor pathological changes [22-23].

\section{Cassia occidentalis (Kasmarda)}

The nephroprotective activity of the $70 \%$ hydroalcoholic extract of Cassia occidentalis was tested against gentamicin induced nephrotoxicity in rats.[24-26]

\section{Casuarina equisetifolia}

The nephroprotective activity of methanolic extract of Casuarina equisetifolia leaves was studied in gentamicin induced nephrotoxicity in Wistar rats. Casuarina equisetifolia leaves extract ameliorates gentamicin-induced nephrotoxicity and oxidative damage by scavenging oxygen free radicals, decreasing lipid peroxidation and improving intracellular antioxidant defense [27-28].

\section{Citrullus colocynthis}

The nephropathy protective effect of Citrullus colocynthis fruits extract was studied in streptozotocin induced diabetes in rats. The study clearly demonstrated that Citrullus colocynthis fruit exerted protective effects on the kidney functions and tissues. So it may play a role in prevent nephropathy as one of microvascular complications of diabetes mellitus [25-26].

\section{Crocus sativus (Kumkum)}

The protective effects of saffron extract and crocin was evaluated in chronic stress induced oxidative stress damage of the brain, liver and kidneys in rats. Chronic stress, the levels of the lipid peroxidation product, malondialdehyde (MDA), the total antioxidant reactivity (TAR), as well as antioxidant enzyme activities glutathione peroxidase (GPX), glutathione reductase (GR) and superoxide dismutase (SOD) were measured in the brain, liver and kidneys tissues after the end of chronic stress[2930]

\section{Cuminum cyminum (Jeeraka)}

The effect of Cuminum cyminum (Cumin) on kidney exposed to profenofos was evaluated in female swiss albino mice. The results showed that cumin was effective in normalizing the uric acid and creatinine level [31-32].

\section{Cymbopogon schoenanthus}

Cymbopogon scheonanthus extract has prophylactic effect in oxalate stone formation [35-36].

\section{Cynodon dactylon (Durva)}

Cynodon dactylon extract reduced the levels of calcium oxalate deposition especially in medullary and papillary sections from of the kidney of the treated rats [35].

\section{Daucus carota (Garjar)}

The renoprotective activity of Daucus carota root extract was studied in renal ischemia reperfusion injury in rats. Daucus carota extracts was associated with a significantly lower malondialdehyde level. 
Accordingly, Daucus carota extracts exerted renoprotective activity probably by the free radical scavenging activity [36-37].

\section{Foeniculum vulgare (Mishreya)}

The aqueous extract of Foeniculum vulgare seeds, Solanum nigrum fruit and their mixture significantly prevented renal damage by normalizing increased levels of renal markers. Mixture of both plants at high doses exhibited improved nephroprotective and antioxidant activities [38].

\section{Glycyrrhiza glabra (Yeshtimadhu)}

Polyuria in rats with gentamicin-induced acute renal failure was associated with down-regulation of renal aquaporin 2 in the inner and outer renal medulla, and cortex. Glycyrrhizin (200 mg/kg/day) administration restored the expression of aquaporin 2 with paralleled changes in urine output. The changes in renal functional parameters (creatinine clearance, urinary osmolality, and solutefree reabsorption), accompanying acute renal failure were also partially restored after administration of glycyrrhizin. Histological changes in rats with gentamicin-induced acute renal failure were also abrogated by glycyrrhizin treatment [39].

Table 2: Research Work Done On Nephro-protective Drugs[41]

\begin{tabular}{|c|c|c|c|c|c|c|}
\hline $\begin{array}{l}\mathrm{Sr} \\
\mathrm{No}\end{array}$ & Botanical name & Family & $\begin{array}{l}\text { Part } \\
\text { used }\end{array}$ & $\begin{array}{l}\text { Chemical } \\
\text { constituents }\end{array}$ & $\begin{array}{l}\text { Screening } \\
\text { method }\end{array}$ & References \\
\hline 1 & $\begin{array}{l}\text { Aerva lanata } \\
\text { (Gorakshganja) }\end{array}$ & $\begin{array}{l}\text { Rutacea } \\
\text { e }\end{array}$ & $\begin{array}{l}\text { Whole } \\
\text { plant }\end{array}$ & $\begin{array}{l}\text { Botulin, } \beta \text {-sitosterol, } \\
\text { Amyrin, } \\
\text { Hentriacontane, } \\
\text { Campesterol, } \\
\text { Stigma sterol, } \\
\text { Kaempferol, } \\
\text { Propionic acid, } \beta \text { - } \\
\text { carboline-I, } \\
\text { Aervoside and } \\
\text { Aervolanine }\end{array}$ & $\begin{array}{l}\text { Gentamyci } \\
\mathrm{n} \text { induced }\end{array}$ & $\begin{array}{l}\text { Paller et.al., } \\
19904\end{array}$ \\
\hline 2 & $\begin{array}{l}\text { Crataeva } \\
\text { nurvula } \\
\text { (Varuna) }\end{array}$ & $\begin{array}{l}\text { Cappari } \\
\text { daceae }\end{array}$ & Fruit & $\begin{array}{l}\text { Kaemferol-3-O-a- } \\
\text { D-glucoside, } \\
\text { Quercitin-3-O-a-D- } \\
\text { glucoside, } \\
\text { Flavanoids, } \\
\text { Glucosinolates, } \\
\text { Steroids, Lupeol } \\
\text { and Tannins }\end{array}$ & $\begin{array}{l}\text { Gentamyci } \\
\text { n induced }\end{array}$ & $\begin{array}{l}\text { Kore et.al., } \\
20114\end{array}$ \\
\hline 3 & $\begin{array}{l}\text { Orthosiphon } \\
\text { stamineus } \\
\text { (Java tea) }\end{array}$ & $\begin{array}{l}\text { Laminac } \\
\text { eae }\end{array}$ & $\begin{array}{l}\text { Whole } \\
\text { plant }\end{array}$ & $\begin{array}{l}\text { Flavanoids, } \\
\text { Phenols, } \\
\text { Carbohydrates, } \\
\text { Steroids, Tannins, } \\
\text { Glycosides, Terpins } \\
\text { and Saponins }\end{array}$ & $\begin{array}{l}\text { Gentamyci } \\
\mathrm{n} \text { induced }\end{array}$ & $\begin{array}{l}\text { Kannapan } \\
\text { et.al.,20104 } \\
1\end{array}$ \\
\hline 4 & $\begin{array}{l}\text { Strychnos } \\
\text { potatorum }\end{array}$ & $\begin{array}{l}\text { Logania } \\
\text { ceae }\end{array}$ & Seed & $\begin{array}{l}\text { Flavanoids, } \\
\text { Phenols, Saponins, } \\
\text { Alkaloids, Steroids, } \\
\text { Tannins, }\end{array}$ & $\begin{array}{l}\text { Gentamyci } \\
\mathrm{n} \text { induced }\end{array}$ & $\begin{array}{l}\text { Ruby } \\
\text { Varghese } \\
\text { et.al., } \\
201142\end{array}$ \\
\hline
\end{tabular}




\begin{tabular}{|c|c|c|c|c|c|c|}
\hline & & & & $\begin{array}{l}\text { Glycosides, and } \\
\text { Lignins }\end{array}$ & & \\
\hline 5 & Aerva javanica & $\begin{array}{l}\text { Amarant } \\
\text { haceae }\end{array}$ & $\begin{array}{l}\text { Fresh } \\
\text { roots }\end{array}$ & $\begin{array}{l}\text { Isoquercetin, } 5 \\
\text { methylmellein, 2- } \\
\text { hydroxy-3-O- } \beta \text { - } \\
\text { primeveroside } \\
\text { naphthalene-1,4- } \\
\text { dione, Apigenin7- } \\
\text { Oglucoronide } \\
\text { and Kaempferol }\end{array}$ & $\begin{array}{l}\text { Cisplatin } \\
\text { induced }\end{array}$ & $\begin{array}{l}\text { Vinit } \\
\text { movaliya } \\
\text { et.al., } \\
201143\end{array}$ \\
\hline 6 & $\begin{array}{l}\text { Carica papaya } \\
\text { (Papita) }\end{array}$ & $\begin{array}{l}\text { Caricace } \\
\text { ae }\end{array}$ & Seed & $\begin{array}{l}\text { Flavanoids, } \\
\text { Phenols, Alkaloids, } \\
\text { Protein, Sterols, } \\
\text { Terpenoids, } \\
\text { Carbohydrates, } \\
\text { Steroids, Tannins, } \\
\text { Glycosides, Terpins } \\
\text { and Saponins }\end{array}$ & $\begin{array}{l}\text { Cisplatin } \\
\text { induced }\end{array}$ & $\begin{array}{l}\text { Subal } \\
\text { debnath } \\
\text { et.al., } \\
201044\end{array}$ \\
\hline 7 & $\begin{array}{l}\text { Ficus religiosa } \\
\text { L } \\
\text { (Vata) }\end{array}$ & $\begin{array}{l}\text { Moracea } \\
\text { e }\end{array}$ & Latex & $\begin{array}{l}\text { Flavonoids, Amino } \\
\text { acids and Tannins }\end{array}$ & $\begin{array}{l}\text { Cisplatin } \\
\text { induced }\end{array}$ & $\begin{array}{l}\text { Yogesh } \\
\text { chand yadav } \\
\text { et.al., } 2011\end{array}$ \\
\hline 8 & $\begin{array}{l}\text { Pedalium murex } \\
\text { Linn } \\
\text { (Brihata } \\
\text { Gokshura) }\end{array}$ & $\begin{array}{l}\text { Pedaliac } \\
\text { eae }\end{array}$ & $\begin{array}{l}\text { Dried } \\
\text { fruits }\end{array}$ & $\begin{array}{l}\text { Flavanoids, } \\
\text { Flavones, } \\
\text { Alkaloids, } \\
\text { Triterpenoids, } \\
\text { Carbohydrates, } \\
\text { Glycosides and } \\
\text { Saponins. }\end{array}$ & $\begin{array}{l}\text { Cisplatin } \\
\text { induced }\end{array}$ & $\begin{array}{l}\text { Shelke } \\
\text { et.al., } \\
200946\end{array}$ \\
\hline 9 & $\begin{array}{l}\text { Vernonia } \\
\text { cinerea } \\
\text { (Sahadevi) }\end{array}$ & $\begin{array}{l}\text { Compos } \\
\text { itae }\end{array}$ & $\begin{array}{l}\text { Aerial } \\
\text { parts }\end{array}$ & $\begin{array}{l}\text { Triterpenoids like } \\
\alpha \text {-amyrin, } \beta \text {-amyrin } \\
\text { and lupeol }\end{array}$ & $\begin{array}{l}\text { Cisplatin } \\
\text { induced }\end{array}$ & $\begin{array}{l}\text { Sreedevi } \\
\text { et.al., } \\
201147\end{array}$ \\
\hline 10 & $\begin{array}{l}\text { Acorus calamus } \\
\text { (Vacha) }\end{array}$ & Araceae & $\begin{array}{l}\text { Aerial } \\
\text { parts }\end{array}$ & $\begin{array}{l}\text { Monoterpene, } \\
\text { Sesquiterpene, } \\
\text { Phenyl } \\
\text { propanoid, } \\
\text { Flavonoids, } \\
\text { Quinone and } \\
\text { basarone }\end{array}$ & $\begin{array}{l}\text { Acetamino } \\
\text { phen } \\
\text { induced }\end{array}$ & $\begin{array}{l}\text { Palani et.al., } \\
201048\end{array}$ \\
\hline 11 & $\begin{array}{l}\text { Boerhaavia } \\
\text { diffusa } \\
\text { (Punernava) }\end{array}$ & $\begin{array}{l}\text { Nyctagi } \\
\text { naceae }\end{array}$ & Root & $\begin{array}{l}\text { Flavonoids, } \\
\text { Alkaloids, Steroids, } \\
\text { Triterpenoids, } \\
\text { Lipids, Lignins, } \\
\text { carbohydrates, } \\
\text { Proteins and } \\
\text { Glycoproteins }\end{array}$ & $\begin{array}{l}\text { Acetamino } \\
\text { phen } \\
\text { induced }\end{array}$ & $\begin{array}{l}\text { Surendra } \\
\text { et.al., } \\
201149\end{array}$ \\
\hline 12 & $\begin{array}{l}\text { Indigofera } \\
\text { barberi L } \\
\text { (Neela) }\end{array}$ & $\begin{array}{l}\text { Fabacea } \\
\mathrm{e}\end{array}$ & $\begin{array}{l}\text { Whole } \\
\text { plant }\end{array}$ & $\begin{array}{l}\text { Flavonoids, } \\
\text { Phenolic acid and } \\
\text { sterols }\end{array}$ & $\begin{array}{l}\text { Acetamino } \\
\text { phen } \\
\text { induced }\end{array}$ & $\begin{array}{l}\text { Palani et.al., } \\
200850\end{array}$ \\
\hline 13 & \begin{tabular}{|l|} 
Pimpinella \\
tirupatiensis
\end{tabular} & $\begin{array}{l}\text { Apiacea } \\
\mathrm{e}\end{array}$ & $\begin{array}{l}\text { Whole } \\
\text { plant }\end{array}$ & $\begin{array}{l}\text { Alkaloids, } \\
\text { Flavonoids, }\end{array}$ & $\begin{array}{l}\text { Acetamino } \\
\text { phen }\end{array}$ & $\begin{array}{l}\text { Palani et.al., } \\
200951\end{array}$ \\
\hline
\end{tabular}




\begin{tabular}{|c|c|c|c|c|c|c|}
\hline & & & & $\begin{array}{l}\text { Flavones, } \\
\text { Volatile oils, } \beta \text { - } \\
\text { Bisaboline, } \Delta-3 \text { - } \\
\text { Carene, } \\
\text { Cis-Carveol, } \\
\text { Enemol, } \Delta \text {-Carveol } \\
\text { and } \\
\text { Methylgeranate }\end{array}$ & induced & \\
\hline 14 & $\begin{array}{l}\text { Curcuma longa } \\
\text { (Haridra) }\end{array}$ & $\begin{array}{l}\text { Zingebe } \\
\text { raceae }\end{array}$ & $\begin{array}{l}\text { Rhizo } \\
\text { me }\end{array}$ & $\begin{array}{l}\text { Curcumin, } \\
\text { Turmeric oil, } \\
\text { Terpenoids, } \\
\text { Curcumin } \\
\text { (Terpene), Starch } \\
\text { and } \\
\text { Albumnoids }\end{array}$ & $\begin{array}{l}\text { Cadmium } \\
\text { induced }\end{array}$ & $\begin{array}{l}\text { Eduardo } \\
\text { Molina- } \\
\text { Jijon et.al., } \\
2011\end{array}$ \\
\hline 15 & $\begin{array}{l}\text { Drynaria } \\
\text { fortune }\end{array}$ & $\begin{array}{l}\text { Polypod } \\
\text { iaceae }\end{array}$ & $\begin{array}{l}\text { Whole } \\
\text { plant }\end{array}$ & $\begin{array}{l}\text { Arsenic, } \quad \mathrm{Ca} 2+, \\
\mathrm{Cu} 2+, \quad \text { Glucose, } \\
\text { Iron, } \\
\mathrm{Mg}, \quad \mathrm{Mn}, \quad \mathrm{Hg}, \\
\text { Naringin, } \mathrm{K}+, \mathrm{Na}+, \\
\text { Starch and Zinc }\end{array}$ & $\begin{array}{l}\text { Silver } \\
\text { chloride } \\
\text { induced }\end{array}$ & $\begin{array}{l}\text { Kore et.al., } \\
201153\end{array}$ \\
\hline 16 & $\begin{array}{l}\text { Eruca sativa } \\
\text { (Tuvari) }\end{array}$ & $\begin{array}{l}\text { Crassula } \\
\text { ceae }\end{array}$ & Seeds & Flavanoids & $\begin{array}{l}\text { Mercuric } \\
\text { chloride } \\
\text { induced }\end{array}$ & $\begin{array}{l}\text { Sarwar } \\
\text { Alam et.al., } \\
200754\end{array}$ \\
\hline 17 & $\begin{array}{l}\text { Moringa } \\
\text { oleifera } \\
\text { (Shigru) }\end{array}$ & $\begin{array}{l}\text { Moringa } \\
\text { ceae }\end{array}$ & eds & $\begin{array}{l}\text { Vitamin A, } \\
\text { Nicotinic acid, } \\
\text { Ascorbic } \\
\text { acid, Vitamin B, } \\
\text { Fatty acid, Glucose, } \\
\text { Sucrose, Citric } \\
\text { acid, Malic acid, } \\
\text { Succinic acid, } \\
\text { Fumaric acid and } \\
\text { Oxalic acid }\end{array}$ & $\begin{array}{l}\text { Fluoride } \\
\text { induced }\end{array}$ & $\begin{array}{l}\text { Ranjan } \\
\text { et.al., } \\
200955\end{array}$ \\
\hline 18 & $\begin{array}{l}\text { Tamarindus } \\
\text { indica } \\
\text { (Chincha) }\end{array}$ & $\begin{array}{l}\text { Caesalpi } \\
\text { naceae }\end{array}$ & $\begin{array}{l}\text { Fruit } \\
\text { pulp }\end{array}$ & $\begin{array}{l}\text { Polysaccharides, } \\
\text { Balsamine, } \\
\text { Catechin, } \\
\text { Nasturtium, } \\
\text { Tamarin, } \\
\text { Phosphatidic } \\
\text { acid, Phosphatidic } \\
\text { choline, } \\
\text { Ethanollamine, } \\
\text { Serine, Inositol, } \\
\text { Alkaloid, Citric } \\
\text { acid, Tartaric acid } \\
\text { and Pottasiumbitartrate }\end{array}$ & $\begin{array}{l}\text { Fluoride } \\
\text { induced }\end{array}$ & $\begin{array}{l}\text { Ranjan } \\
\text { et.al., } \\
200955\end{array}$ \\
\hline 19 & $\begin{array}{l}\text { Tectona grandis } \\
\text { (Shaka) }\end{array}$ & $\begin{array}{l}\text { Verbana } \\
\text { ceae }\end{array}$ & Bark & $\begin{array}{l}\text { Lapachol, Dehydro- } \\
\alpha \text {-lapachone, } \\
\text { Methyl quinizarin } \\
\text { and Squalene. }\end{array}$ & $\begin{array}{l}\text { Alloxan } \\
\text { induced }\end{array}$ & $\begin{array}{l}\text { Ghasias et. } \\
\text { al., } 201056\end{array}$ \\
\hline
\end{tabular}




\begin{tabular}{|c|c|c|c|c|c|c|}
\hline 20 & Ginkgo biloba & $\begin{array}{l}\text { Ginkgoa } \\
\text { ceae }\end{array}$ & $\begin{array}{l}\text { Whole } \\
\text { plant }\end{array}$ & $\begin{array}{l}\text { Flavonoids, } \\
\text { Bilobalide, } \\
\text { GingkolideA, } \\
\text { Gingkolide B and } \\
\text { Gingkolide } \\
\text { CandBiflanoide }\end{array}$ & $\begin{array}{l}\text { Streptozoto } \\
\text { cin induced }\end{array}$ & $\begin{array}{l}\text { Welta et.al., } \\
200757\end{array}$ \\
\hline 21 & $\begin{array}{l}\text { Abutilon } \\
\text { indicum } \\
\text { (Atiibala) }\end{array}$ & $\begin{array}{l}\text { Malvace } \\
\text { ae }\end{array}$ & $\begin{array}{l}\text { Whole } \\
\text { plant }\end{array}$ & $\begin{array}{l}\text { Saponins, } \\
\text { Flavonoids and } \\
\text { Tannins. }\end{array}$ & $\begin{array}{l}\text { Gentamicin } \\
\text { induced }\end{array}$ & $\begin{array}{l}\text { Kakasaheb } \\
\text { Khore et.al., } \\
201158\end{array}$ \\
\hline 22 & $\begin{array}{l}\text { Euphorbia } \\
\text { neriifolia } \\
\text { (Snuhi) }\end{array}$ & $\begin{array}{l}\text { Euphorb } \\
\text { iaceae }\end{array}$ & Leaves & $\begin{array}{l}\text { Saponins, } \\
\text { Flavonoids and } \\
\text { Tannins }\end{array}$ & $\begin{array}{l}\mathrm{N} \text {-nitroso } \\
\text { dimethyl } \\
\text { amine } \\
\text { induced }\end{array}$ & $\begin{array}{l}\text { Pracheta } \\
\text { et.al., } \\
201159\end{array}$ \\
\hline 23 & $\begin{array}{l}\text { Rubia cardifolia } \\
\text { Linn } \\
\text { (Manjishtha) }\end{array}$ & $\begin{array}{l}\text { Rubiace } \\
\text { ae }\end{array}$ & Root & $\begin{array}{l}\text { Purpurin, Manjistin, } \\
\text { Garancin, } \\
\text { Purpuroxanthin, } \\
\text { Resin, Glucose, } \\
\text { Sucrose, } \\
\text { Triterpenes, } \\
\text { Lucidine, } \\
\text { Anthroquinine, } \\
\text { Fattyacids and } \\
\text { Gum. }\end{array}$ & $\begin{array}{l}\text { Ethylene } \\
\text { glycol } \\
\text { induced }\end{array}$ & $\begin{array}{l}\text { Kalyani } \\
\text { Divakar et. } \\
\text { al., } 201060\end{array}$ \\
\hline $\begin{array}{l}2 \\
4\end{array}$ & $\begin{array}{l}\text { Punicagranatu } \\
m L \\
\text { (Dadima) }\end{array}$ & $\begin{array}{l}\text { Puniace } \\
\text { ae }\end{array}$ & $\begin{array}{l}\text { Fruit } \\
\text { peel }\end{array}$ & $\begin{array}{l}\text { Ellagic } \\
\text { Ellagitannins acid, } \\
\text { gallic } \\
\text { acid. }\end{array}$ & $\begin{array}{l}\text { Ferric } \\
\text { nitrilo tri } \\
\text { acetate } \\
\text { induced }\end{array}$ & $\begin{array}{l}\text { Mahgoub } \\
\text { Mohammed } \\
\text { AHMED } \\
\text { et.al., } \\
201061\end{array}$ \\
\hline
\end{tabular}

\section{Observation and Discussion-}

The alcoholic and aqueous extracts of the Hybanthus enneaspermus possesses significant curative and preventive nephroprotective activity. According to Acharya charak, mootra dosh vikara is tretised by punarnava, haritaki, shunthi, nagarmotha, shilajeet, Gokshura, yavakshar etc. Kansharitaki is also very helpful in mootra dosha vikar as well as vata-kaph odema .Shilajeet is helpful with take gomutra in mootra dosha vikar. Tectona grandis has the potential to treat diabetes mellitus and prevent the associated renal damage. Traditionally Tectona grandis is used in treatment of diabetes, lipid disorders, inflammation, ulcer, and bronchitis.
Tectona grandis is reported to have antiulcer, antimicrobial, wound healing, anticancer, and anti-renal damage activity. Chronic kidney disease (CKD) can be prevented or delayed by early treatment using angiotensin IIconverting enzyme inhibitors and angiotensin II-receptor blockers. A great amount of the world's population has been using traditional Chinese herbal medicine (astragalus; angelica; rhubarb) for treatment of CKD. Listed selected plants as like in table used by Aboriginal tribes all over Canada for kidney diseases. Mostly, these are used for diuresis, renal stones and cleansing the kidneys. A brief review of the literature shows different plants being effective in preventing/treating 
renal diseases. Some renal conditions reported to respond to plant therapy are glomerulonephritis, IgA nephropathy, membranous nephropathy, glomerulosclerosis, immune complex nephritis, nephrotic syndrome, lupus, tubule interstitial nephritis, chronic allograft nephropathy, kidney stones etc. Some pharmacological characteristics seen in plants that may contribute in the abovementioned conditions are anti-inflammation; antioxidation. The therapeutic property of Sesamum indicum seeds propitious in improving nephropathy by significantly improving serum parameters and histopathological evidence also suggests the same. The seed contains appreciable amounts of various bioactive components including tocopherols, phytosterols, resveratrol and flavonoids, and the lignans sesamin and sesamolin. The identification and management of early stage diabetic kidney disease is important, but the majority of people exhibit no symptoms until the disease is more advanced renal damage. Hemidesmus indicus was found on study base that aqueous and methanolic extracts have more significant inhibitory effect on salt water feeding induced severity of microalbuminuria, serum urea and creatinine, myocyte diameter and retention of $\mathrm{Na}+$ and water and increases the serum calcium level. It is potent natural nephroprotective also a cardioprotective. Orthosiphon stamineus is the one of the important medicinal plant which used for kidney based Problems traditionally. The ethanolic extracts of $O$. stamineus leaves have been used for the nephroprotective activity. The whole plant of the Orthosiphon stamineus (cats whiskers) is used as gall bladder, kidney, liver, bladder problems, painful tooth, hypertension and genitourinary diseases. Cat's Whiskers is a perennial herb found mainly throughout south East Asia and tropical Australia. The herb is popularly known as Java tea. O. stamineus is used widely in the form of herbal tea among the South East Asian population. Pharmacological properties of Polyphenols are found in the renal area, acting as diuretic, anti-inflammatory, antispasmodic, and antioxidant agents. Various polyphenolic compoundshave been reported for their nephroprotective activity with a good level of renal protection. Therefore, considering the important role of polyphenolic compounds in the prevention or reduction of renal disorders induced by various nephrotoxic chemical agents, in this way, he was summarized the some antioxidant plants, such as, Achyrocline satureioides, Zingiber officinalis, Teminalia chebula etc having Nephroprotective properties .Solanum nigrum was found that renal markers (urea.serum creatnine, uric acid) were brought back to normal. Thus it is inferred that Solanum nigrum preserves the functional capacity of the kidney against ethanol toxicity. Launaea procumbens effectively protect kidneys as well as decreased Serum level of creatinine, urobilinogen, BUN, direct bilirubin, total bilirubin and globulin while total proteins, albumin, through antioxidant and free radical scavenging effects of flavonoids and saponins.

\section{Conclusion-}

From this study, it is clear that the medicinal plants play a prominent role against various diseases. A variety of medicinal plants and plants extracts have been reported for its significant nephroprotective activity in animal models. The nephroprotective activity is probably due to the presence of Flavanoids in all the few medicinal plants. The results of this study indicate that extracts of leaves and plants of some medicinal plants have good potentials for use in kidney damages.

\section{Reference -}


[1] Porter GA and Bennett WM. Nephrotoxic acute renal failure due to common drugs American journal of Physiology, 1981; 241 (7): F1-F8

[2] Hoitsma AJ, Wetzels JF and Koene RA. Drug induced nephrotoxicity. Aetiology, clinical features and management, Drug Saf, 1991; 6 (2): 131-147.

[3] Paller MS., Drug induced nephropathies Med Clin North Am, 1990; 74 (4):909-917.

[4] http://farmacists.blogspot.com

[5] Herfindal, Gourley. Text book of therapeutic drug and disease management. 7th Edn. Charcil Livingstone, London; 2000; 425-36.

[6] Barry M, Brenner, Floyd C, Rector. The kidney 6th Ed. Vol I, W.B. Saunders Company, Philadelphia; 2000; 3-67.

[7] Agnivesa's Caraka Samhita Text with English translation \& critical exposition based on Chakrapani Datta's

Ayurveda Dipika By Dr. Ram Karan Sharma and Vaidya Bhagvan Dash, Volume II, Published by Choukhamba

Sanskrit Series Office, Varanasi, Edition Reprint, Vimana Sthan, Chapter 5, Slok no 20, 2010; 179.

[8] Agnivesa's Caraka Samhita Text with English translation \& critical exposition based on Chakrapani Datta's

Ayurveda Dipika By Dr. Ram Karan Sharma and Vaidya Bhagvan Dash, Volume II, Published by Choukhamba Sanskrit Series Office, Varanasi, Edition Reprint nidana Sthan, Chapter 4, Slok no $8,2010,56$

[9] A REVIEW ON MEDICINAL PLANTS FOR NEPHROPROTECTIVE ACTIVITY MOHANA LAKSHMI. S*, USHA KIRAN REDDY. T AND SANDHYA RANI. KS Department of Pharmacognosy, Sree Vidyanikethan
College of Pharmacy, Tirupati-517102, Andhra Pradesh, India, Vol 5, Issue 4, 2012

[10] Sharma RK, Rajani GP, Sharma V and Komala N. (2011). Effect of ethanolic and aqueous extracts of Bauhinia variegata Linn. on gentamicin-induced nephrotoxicity in rats. Indian Journal Pharmaceutical Education Research, 45(2), 192-198

[11] Al-Snafi AE. (2013). The Pharmacological importance of Bauhinia variegata. A Review. Journal of Pharma Sciences and Research, 4(12), 160-164.

\section{[12] Mingyu D, Mingzhang L, Quihong $Y$,} Weiming $U$, Jianxing $X$ and Weinming $X$. (1995). A study on Benincasa hispida contents effective for protection of kidney. Jiangsu Journal Agriculture Science, 11, 46-52.

[13] Al-Snafi AE. (2013). The Pharmacological Importance of Benincasa hispida. A review. International Journal of Pharma Sciences and Research, 4(12), 165-170

[14] Evaluation of Nephro-protective and Anti-Nephro toxicity in Rakta punernava and Gokshura by Kulkarni yogini Ramchandra

[15] Rajamurugan R, Suyavaran A, Selvaganabathy $\mathrm{N}$, Ramamurthy $\mathrm{CH}$, Reddy GP, Sujatha $V$ and Thirunavukkarasu C.(2012). Brassica nigra plays a remedy role in hepatic and renal damage. Pharmaceutical Biology, 50(12), 1488-1497.

[16] Al-Snafi AE. (2015). The pharmacological importance of Brassica nigra and Brassica rapa grown in Iraq. Journal of Pharmceutical Biology, 5(4), 240-253 
[17] Kim YH, Kim YW, Oh YJ, Back NI, Chung SA, Chung HG, Jeong TS, Choi MS and Lee KT. (2006). Protective effect of the ethanol extract of the roots of Brassica rapa on cisplatin-induced nephrotoxicity in LLC-PK 1 cells and rats. Biological Pharmaceutical Bulletin, 29(12), 2436-2441

[18] Majaz QA, Tatiya AU, Khurshid M and Nazim S. (2011). The miracle plant (Kalanchoe pinnata): A photochemical and pharmacological review. International Journal of Research in Ayurveda and Pharmacy, 2(5), 1478-1482

[19] Harlalka GV and Patil CR. (2007). Protective effect of Kalanchoe pinnata pers. (Crassulaceae) on Gentamycin induced nephrotoxicity in rats. Indian Journal of Pharmaccology, 39(4), 201205.

[20] Patil R, Bhargava K, Patel P, Singh K and Surana J. (2008). Diuretic and anti urolthiatic activity of hydroalcoholic extracts of leaves of Kalanchoe pinnata pers. Journal of Pharmaceutical Research, 7(2), 87-91.

[2] 1 Al-Snafi AE. (2013). The Chemical constituents and pharmacological effects of Bryophyllum calycinum- A review. Journal of Pharma Sciences and Research, 4(12), 171-176.

[22] Abou El-Soud N H, El-Lithy N A, ElSaeed G, Wahby M S, Khalil M Y, Morsy F and Shaffie N. (2014). Renoprotective effects of caraway (Carum carvi L.) essential oil in streptozotocin induced diabetic rats. Journal of Applied Pharmaceutical Science, 4(02), 027-033.

[23] Al-Snafi AE. (2015). The chemical constituents and pharmacological effects of Carum carvi - A review. Indian Journal of Pharmaceutical Science and Research, 5(2), 72-82.
[24] Kumar AR and Abbulu K. (2011). Antioxidant activity of ethanolic extract of Cassia occidentalis against carbon tetrachloride induced oxidative stress in Wistar rats. International Journal Chemistry Science, 9(1), 378-386.

\section{[25] Gowrisri M, Kotagiri S, Vrushabendra} SBM, Archana SP and Vishwanath KM. (2012). Anti-oxidant and nephroprotective activities of Cassia occidentalis leaf extract against gentamicin induced nephrotoxicity in rats. Research Journal of Pharmaceutical, Biological and Chemical Sciences, 3(3), 684-694.

[26] Al-Snafi AE. (2015). The therapeutic importance of Cassia occidentalis - An overview.Indian Journal of Pharmaceutical Science \& Research, 5(3), 158-171.

[27] El-Tantawy WH, Mohamed SA and Abd Al Haleem EN. (2013). Evaluation of biochemical effects of Casuarina equisetifolia extract on gentamicininduced nephrotoxicity and oxidative stress in rats. Phytochemical analysis. Journal Clinical Biochemistry Nutrition, 53(3), 158-165.

[28] Al-Snafi AE. (2015). The pharmacological importance of Casuarina equisetifolia - An overview. InternationaJournal of Pharmacological Screening Methods, 5(1), 4-9.

[29] Bandegi AR, Rashidy-Pour A, Vafaei AA and Ghadrdoost B. (2014). Protective effects of Crocus sativus $L$ extract and crocin against chronic-stress induced oxidative damage of brain, liver and kidneys in rats. Advance Pharmaceutical Bulletin, 4(Suppl 2), 493-499.

[30] Al-Snafi AE. (2016). The pharmacology of Crocus sativus- A review. International Organization of Scientific Research: Journal of Pharmacy, 6(6), 8-38. 
[31] Kumar A, Singh JK, Ali M, Kumar R, Kumar A, Nath A, Roy AK, Roy SP and Singh JK. (2011). Evaluation of Cuminum cyminum and Coriandrum sativum on profenofos induced nephrotoxicity in Swiss albino mice. Elixir Applied Botany, $39,4771-4773$.

[32] Kumar R, Ali M, Kumar A. (2014). Nephroprotective effect of Cuminum cyminum on chloropyrifos induced kidney of mice. Adv J Pharm Life sci Res, 2(4), 4653.

[33] Al Haznawi AM, AttarAS, Abdulshakoor AA and Ramadan MA. (2007). Inhibition of calcium oxalate nephrotoxicity with Cymbopogon schoenanthus (Al-Ethkher). MSc thesis. Faculty of Applied Medical Sciences, Saudi Arabia.

[34] Al-Snafi AE. (2016). The chemical constituents and pharmacological activities of Cymbopagon schoenanthus: A review. Chemistry Research Journal, $1(5), 53-61$.

[35] Mousa-Al-Reza H, Rad AK, Rajaei Z, Sadeghian $M H$, Hashemi $N$ and Keshavarzi Z. (2011). Preventive effect of Cynodon dactylon against ethylene glycol-induced nephrolithiasis in male rats. Avicenna Journal Phytomedicine, $1(1), 14-23$

[36] Mital PR, Laxman PJ and Ramesshvar

PK. (2011). Protective effect of Daucus carota rootextract against ischemia reperfusion injury in rats. Pharmacology, 1 , 432-439.

[37] Al-Snafi AE. (2017). Nutritional and therapeutic importance of Daucus carota- A review. International Organization of Scientific Research: Journal of Pharmacy, 7(2), 72-88

[38] Al-Snafi AE. (2018).The chemical constituents and pharmacological effects of Foeniculum vulgare - A review. International Organization of Scientific Research: Journal of Pharmacy, 8(5), 8196.

[39] Zhao H, Zhao M, Wang $Y$, Li F and Zhang Z. (2016). Glycyrrhizic acid attenuates sepsis-induced acute kidney injury by inhibiting NF-KB signaling pathway.

Evidence-Based Complementary and Alternative Medicine,

[40] A Critical review on commonly used herbal drugs in CKD Gupta Mahesh Chand, Trilok chand Journal of Medicinal Plants Studies 2015; 3(4): 44-47

[41] A REVIEW ON MEDICINAL PLANTS FOR NEPHROPROTECTIVE ACTIVITY MOHANA LAKSHMI. S*, USHA KIRAN REDDY. T AND SANDHYA RANI. KS Department of Pharmacognosy, Sree Vidyanikethan College of Pharmacy, Tirupati-517102, Andhra Pradesh, India, Vol 5, Issue 4, 2012 